\title{
Effect of flow field distribution on the synthesis of sub-micron ZSM-5 molecular sieve in a quasi-solid system
}

\author{
Xia Zhi' ${ }^{1}$ Hu Si ${ }^{2}$, Zhang Huan' ${ }^{2}$ Li Xiaofeng ${ }^{1}$, Gong Yanjun² and Dou Tao ${ }^{1,2 *}$ \\ ${ }^{1}$ Taiyuan University of Technology, Taiyuan Shanxi 030024, China \\ ${ }^{2}$ China University of Petroleum, Beijing 102249, China \\ (C) China University of Petroleum (Beijing) and Springer-Verlag Berlin Heidelberg 2013
}

\begin{abstract}
Scale-up synthesis of sub-micron ZSM-5 molecular sieve in a quasi-solid system was investigated. Compared with traditional hydrothermal synthesis, the synthesis in a quasi-solid system has the advantages of high yield, short crystallization time, low energy consumption as well as low emissions. However, the high solid content in the quasi-solid system can cause the mass and heat transfer problems and make scalable production difficult. In order to solve the problem, we have developed a method for the optimization of the mass and heat transfer. By this method one can vary the flow field in the reactor by changing the stirrer speed. Scale-up synthesis of the sub-micron ZSM-5 molecular sieve in a quasi-solid system was carried out in a $5 \mathrm{~L}$ reactor with double propeller-type agitators. The process was investigated with product characterization using X-ray diffraction (XRD) and scanning electron microscopy (SEM) and the flow field information was collected using laser Doppler velocimetry (LDV). The results showed that the flow field patterns can be tuned by using different stirrer speeds, the morphology and size of assynthesized of ZSM-5 can be effectively controlled.
\end{abstract}

Key words: Quasi-solid system, sub-micron ZSM-5, scale-up, stirrer speed, flow field characteristics

\section{Introduction}

ZSM-5 zeolite had been widely investigated by many researchers since it was first reported by the Mobil group in 1978 (Kokotailo et al, 1978). It possesses a unique twodimensional 10-ring channel system and shows favorable thermal stability, acidity, and shape-selectivity. Therefore, much effort has been devoted to its synthesis and application (Yamamura et al, 1994; Sang et al, 2004; Wang et al, 2007; Mohamed et al, 2005; Minkee et al, 2009). ZSM-5 zeolite had been successfully used in catalysis (Chem and Garwood,1978; Wallenstein and Harding, 2001), sorptionseparation as well as ion-exchange applications. Its extensive application potential greatly promoted its production in a commercial scale (Casci, 2005).

Our group described the synthesis of a molecular sieve in a quasi-solid system for the first time (Dou et al, 2008). The synthesis of the molecular sieve in the quasi-solid system has the advantages of high yield, short crystallization time, low energy consumption as well as lower emission than conventional hydrothermal synthesis. However, the high solid content in a quasi-solid system makes the mass transfer

*Corresponding author. email: dtao1@163.com

Received August 16, 2012 and heat transfer more difficult than in the conventional hydrothermal synthesis system. Therefore, the scale-up in the quasi-solid system is more difficult. The scale-up effect is mainly caused by the temperature gradient, concentration gradient, and residence time of the reactant in different scale reactors. To solve scale-up problem in the synthesis of molecular sieves, many studies have been conducted aiming at changing reactants and crystallization time (Xie et al, 2006). Laser Doppler velocimetry (LDV) has proven to be more accurate in the measurement of the flow field in the stirred tank reactor than any other technique, since it can provide flow information even in the unsteady and highly turbulent flow regions as well as in the return flow area of the tank without fluid contact (Yianneskis et al, 1987; Chen et al, 2007; Wang and Chen, 2009; Schwingshackl et al, 2009). In this work, scale-up synthesis of sub-micron ZSM-5 molecular sieve in a quasi-solid system was carried out in a $5 \mathrm{~L}$ reactor with double propeller-type agitators. For the first time, the crystallization process was controlled by tuning the flow field pattern in the reactor by changing the stirrer speed. The process of the scale-up synthesis in the quasi-solid system was investigated with product characterization using X-ray diffraction (XRD) and scanning electron microscopy (SEM) and the flow field information was collected by laser Doppler velocimetry (LDV). 


\section{Experimental}

\subsection{Flow field in a stirred tank}

The three-dimensional flow field and the frequently occurring unstable turbulence complicate the measurement in a stirred tank. In the early stage, the flow field information was obtained by using a pitot tube, electromagnetic velocimeter, piezoelectric probe or a hot film anemometer. However, due to interference by the inserted probe, the flow field information obtained by these methods was not very accurate. The measurement of the flow field in a stirred tank was carried out by using an IFA755 laser Doppler velocimeter (LDV) from TSI Company (USA). A standard LDV system supplies the mean velocity at a single point, and the whole flow field in the stirred tank should be determined by multiple spot measurements (Bolinder and Sundén, 1995; Schäfer et al, 1997). The set-up of the test rig is shown in Fig. 1 .

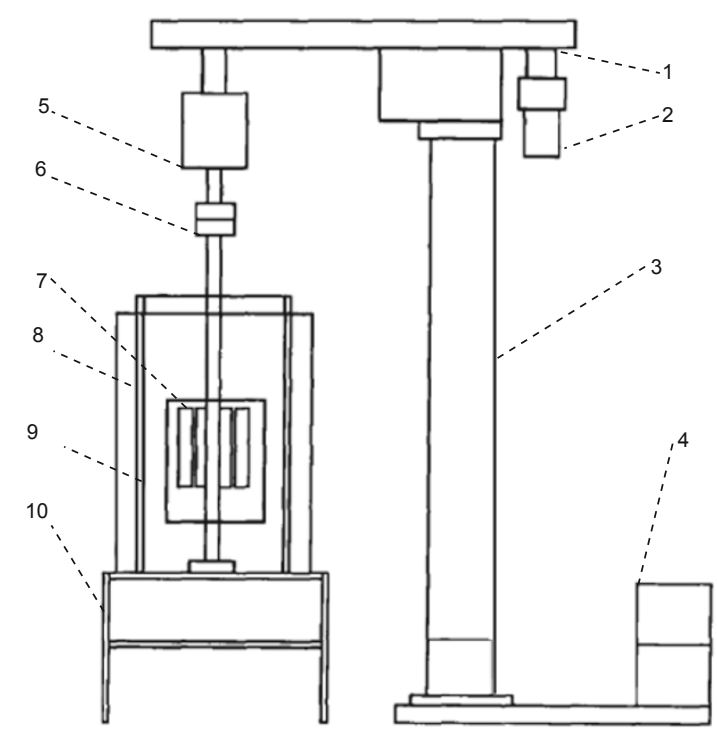

Fig. 1 Test set-up of propeller-type agitator flow

1-Reduction box; 2-Electromagnetic variable-speed motor; 3-Elevator mechanism; 4-Oil station; 5-Sensor; 6-Coupler; 7-Stirrer element; 8-Glass tank; 9-Baffle; 10-Trestle

In this work, described here, double propeller-type agitators were used as the stirrer, with the dimensions of $D=2 T / 3, H=D / 5$ and a clearance of $C=T / 6$ in a cylindrical tank with a diameter $T=150 \mathrm{~mm}$ (as Fig. 2). The thickness of the blades $d=2 \mathrm{~mm}$. The entire measuring circuit was refractive index matched, i.e. the walls were constructed from transparent material with the same refractive index as the model fluid. In addition, the tank was located in a rectangular container filled with the model fluid to eliminate the distorting effect of the rounded surface of the tank on the path of the laser beams.

\subsection{Synthesis}

The reactants were uniformly mixed in the following proportions: $n(\mathrm{Si}): n\left(\mathrm{Al}_{2} \mathrm{O}_{3}\right): n(\mathrm{TPABr}): n\left(\mathrm{Na}_{2} \mathrm{O}\right): n\left(\mathrm{H}_{2} \mathrm{O}\right)$ $=60: 0.19: 15.4: 11: 375$, and then it was transferred to an agitator reactor. The mixture was left and stirred (at stirrer

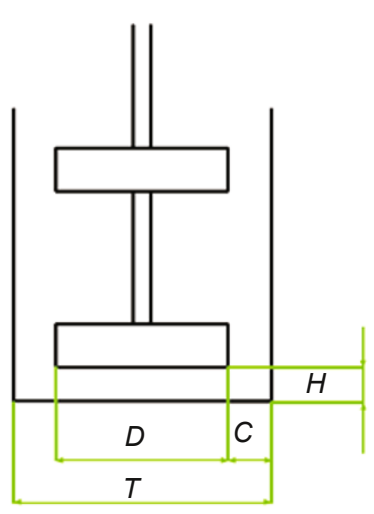

Fig. 2 Dimensions of the propeller-type agitator in the tank

speeds of 200, 400 and $600 \mathrm{r} / \mathrm{min}$ ) in the agitator reactor at $170{ }^{\circ} \mathrm{C}$ for 3 days. The products from the top and the bottom discharge ports were separately filtered, washed thoroughly with de-ionized water and dried at ambient temperature.

\subsection{Characterization}

X-ray diffraction (XRD) measurement was performed by using a Shimadzu XRD-6000 diffractometer $(\mathrm{CuK} \alpha$ radiation, $40 \mathrm{kV}, 30 \mathrm{~mA}$, and scanning angle $5-35^{\circ}$ ). The morphology and crystalline size of the samples were studied by using a Cambridge S-360 scanning electron microscope (SEM) after they were coated with an Au film.

\section{Results and discussion}

\subsection{Flow field patterns at different stirrer speeds}

For a full description of flow structure throughout the stirred tank reactor, the whole flow field was measured at high resolution in the axial direction. Fig. 3 illustrates the flow type under double propeller-type agitators at different stirrer speeds. The arrows represent the non-dimensional velocity. As shown in Fig. 3, the non-dimensional velocity increased significantly with an increase in the stirrer speed. At a stirrer speed of $200 \mathrm{r} / \mathrm{min}$, the flow field was stable, and the fluctuation of the fluid was not violent. At a stirrer speed of $300 \mathrm{r} / \mathrm{min}$, the flow field was similar to that at a stirrer speed of $200 \mathrm{r} / \mathrm{min}$. At a stirrer speed of $400 \mathrm{r} / \mathrm{min}$, the fluctuation of the fluid was more significant than that at a stirrer speed of $300 \mathrm{r} / \mathrm{min}$. At a stirrer speed of $500 \mathrm{r} / \mathrm{min}$, the fluid fluctuation was violent, and the non-dimensional velocity almost disappeared around the stirrer shaft. At a stirrer speed of 600 $\mathrm{r} / \mathrm{min}$, the fluid fluctuation was more violent, and the nondimensional velocity completely disappeared significantly around the stirrer shaft. The reason is that the high tangential velocity of the fluid resulted from high stirrer speed caused the appearance of a whirlpool, and an empty apace of tracer appeared. Fig. 4 shows the tangential velocity distribution at the position $X=50$ of flow at different stirrer speeds under double propeller-type agitators. As shown in the Fig. 4, the tangential velocity increased significantly with an increase in the stirrer speed, at a stirrer speed of $600 \mathrm{r} / \mathrm{min}$, the tangential velocity is much higher than that at the other stirrer speeds. 
Flow type at a stirrer speed of $200 \mathrm{r} / \mathrm{min}$

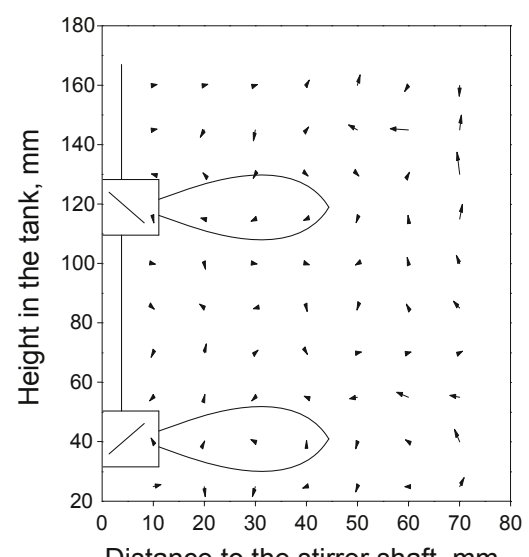

Flow type at a stirrer speed of $400 \mathrm{r} / \mathrm{min}$

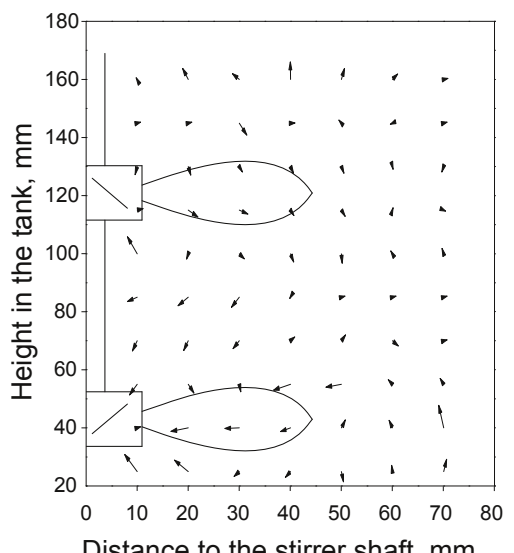

Flow type at a stirrer speed of $300 \mathrm{r} / \mathrm{min}$

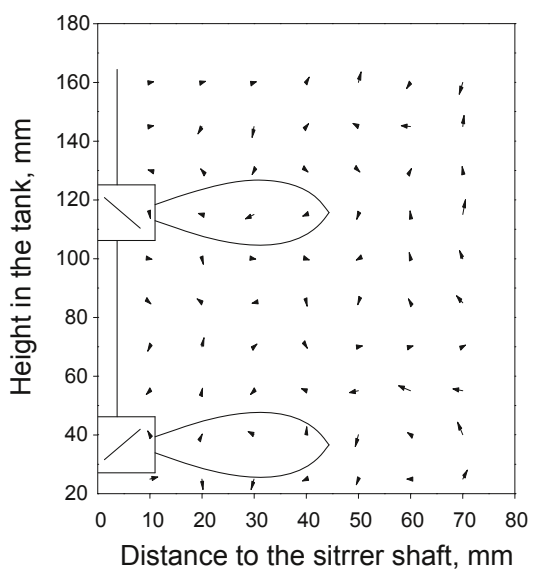

Flow type at a stirrer speed of $500 \mathrm{r} / \mathrm{min}$

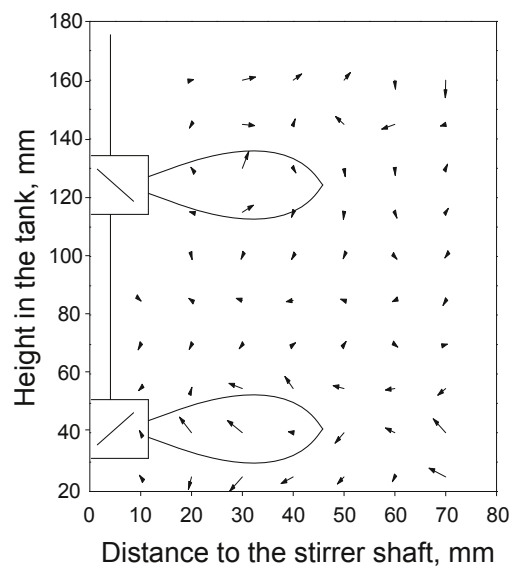

Flow type at a stirrer speed of $600 \mathrm{r} / \mathrm{min}$

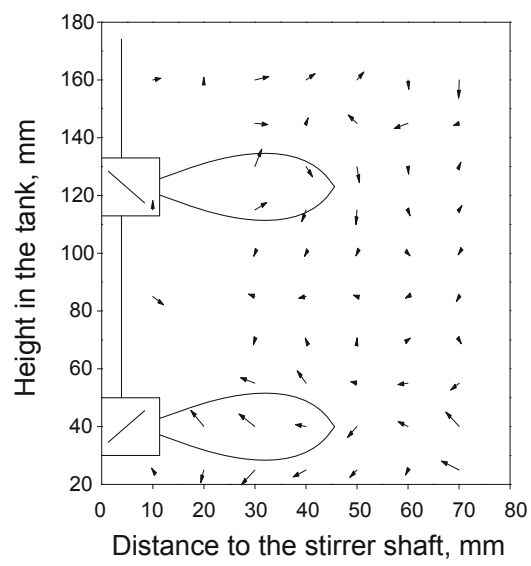

Fig. 3 Flow types of double propeller-type agitators at different stirrer speeds

\subsection{Scale-up synthesis of sub-micron ZSM-5 at different stirrer speeds}

The products sampling from the top and bottom discharge ports at different stirrer speeds were marked as: scs-200-u, scs-200-d, scs-300-u, scs-300-d, scs-400-u, scs-400-d, scs$500-\mathrm{u}, \mathrm{scs}-500-\mathrm{d}$, scs-600-u and scs-600-d (scs-200-u means scale-up sample from the top discharge port at a stirrer speed of $200 \mathrm{r} / \mathrm{min}$; scs-200-d means scale-up sample from the bottom discharge port at a stirrer speed of $200 \mathrm{r} / \mathrm{min}$ ), respectively. The bottom discharge port was located on the side-wall of the reactor bottom.

XRD patterns of the product samples at the stirrer speeds of 200, 300, 400, 500 and $600 \mathrm{r} / \mathrm{min}$ are shown in Fig. 5. The XRD patterns were of typical zeolite ZSM-5. The base lines of the samples at different stirrer speeds were also all quite flat, indicating the as-synthesized products had high relative 


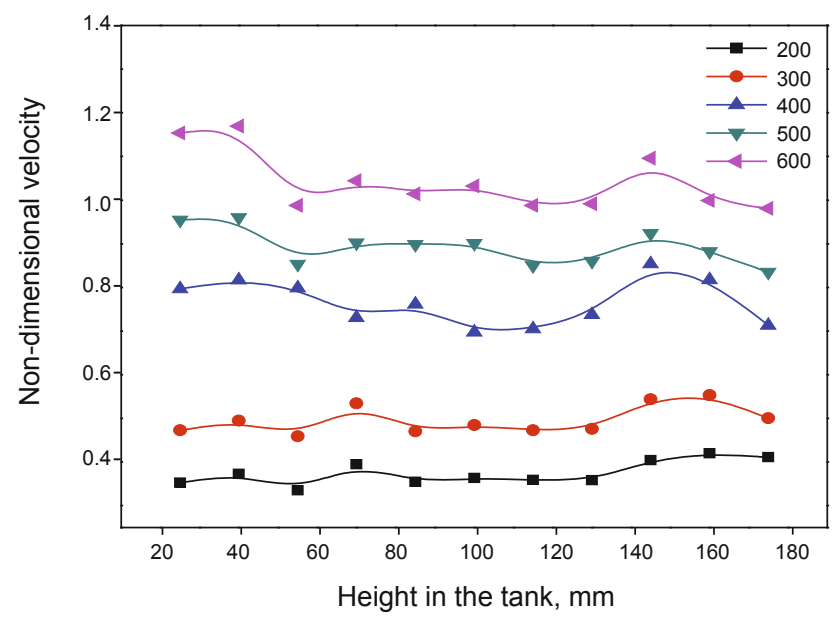

Fig. 4 Tangential velocity distribution of flow at different stirrer speeds under double propeller-type agitators at the axial position of $X=50$

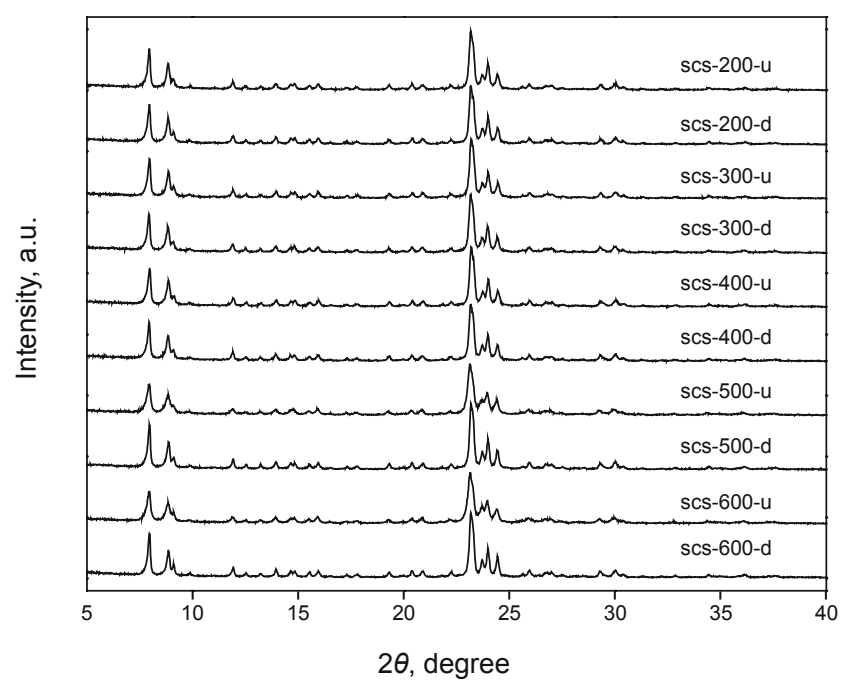

Fig. 5 XRD patterns of the samples from the top and bottom at different stirrer speeds

crystallinity. But small differences exist in the relative crystallinity of the products obtained at different stirrer speeds.

Table 1 shows the relative crystallinity of samples from the top and the bottom at different stirrer speeds. Sample scs$400-\mathrm{u}$ was taken as a standard, and the relative crystallinity of the other samples were calculated by the ratio of the diffraction area to that of the sample scs-400-u. At stirrer speeds of 200 and $300 \mathrm{r} / \mathrm{min}$, the relative crystallinity of the products from the top discharge ports and the bottom discharge ports were all above $90 \%$, while the relative crystallinity was $100 \%$ at a stirrer speed of $400 \mathrm{r} / \mathrm{min}$, and all above $96 \%$ at stirrer speeds of 500 and $600 \mathrm{r} / \mathrm{min}$.

Fig. 6 shows SEM images of the products at different stirrer speeds. The scs-200-u and scs-200-d samples, obtained at a stirrer speed of $200 \mathrm{r} / \mathrm{min}$ were aggregated particles. At a stirred speed of $300 \mathrm{r} / \mathrm{min}$, the samples from the top and bottom discharge port were all dispersed particles, but the crystal sizes were nonuniform. The scs-400-u and scs400-b samples were well dispersed particles with similar
Table 1 Relative crystallinity of the samples from the top and bottom at different stirrer speeds

\begin{tabular}{cccccc}
\hline \multirow{2}{*}{ Sample } & \multicolumn{5}{c}{ Relative crystallinity at different stirrer speeds, \% } \\
\cline { 2 - 6 } & $200 \mathrm{r} / \mathrm{min}$ & $300 \mathrm{r} / \mathrm{min}$ & $400 \mathrm{r} / \mathrm{min}$ & $500 \mathrm{r} / \mathrm{min}$ & $600 \mathrm{r} / \mathrm{min}$ \\
\hline scs-X-u & 97 & 97 & 100 & 100 & 99 \\
scs-X-d & 92 & 94 & 100 & 99 & 98 \\
\hline
\end{tabular}

morphology and uniform crystal sizes. The scs-500-u, scs$500-d$, scs-600-u and scs-600-d samples obtained at stirrer speeds of 500 and $600 \mathrm{r} / \mathrm{min}$ were also well dispersed particles with nonuniform crystal sizes.

As shown in Fig. 7, the crystal ize of scs-200-u sample was $400-800 \mathrm{~nm}$, while scs-200-d was around $1-2 \mu \mathrm{m}$. The crystal size of the scs-300-u sample was 100-200 nm, while scs-300-d was 300-500 nm. The crystal sizes of the samples obtained at a stirred speed of $400 \mathrm{r} / \mathrm{min}$ were both $100 \mathrm{~nm}$. The crystal size of scs-500-u sample was 100-200 nm, while that of scs-500-d was 100-300 nm. The crystal size of scs$600-\mathrm{u}$ sample was $200-300 \mathrm{~nm}$, while that of scs-600-d was 300-500 nm.

Different crystal sizes and morphologies of the samples obtained at different stirrer speeds were related to the fluid field patterns in the reactor. At stirrer speeds of 200 and $300 \mathrm{r} /$ min, the non-dimensional velocity of fluid was very low and could not exert effective fluctuation on the synthesis system with high solid content, thus, the solid reaction mixture would deposit on the bottom of the reactor and could not contact with the reactant in the liquid, resulting in a product with low relative crystallinity. In addition, the heat source of the reactor was located in the side-wall and the bottom of the reactor, thus, a local higher temperature would appear at the bottom of the reactor due to the poor heat transfer of the deposited solid mixture, resulting in the product obtained from the bottom of the reactor having a larger crystal size, as revealed in the SEM results in Fig. 6. In contrast, at the stirrer speed $400 \mathrm{r} / \mathrm{min}$, the non-dimensional velocity was high and the tangential velocity was low, which not only could exert strong fluctuation on the synthesis system and avoid deposition of the solid, but also could make the whole synthesis system in the reactor homogeneous due to the absence of the tangential flow. Hence, the effects of the heat and mass transfer for the scale-up synthesis at this stirrer speed were similar to that for the small scale synthesis and the products from the top and the bottom discharge ports had similar crystal sizes and crystallinity. At stirrer speeds of 500 and $600 \mathrm{r} / \mathrm{min}$, both the non-dimensional velocity of the fluid and the tangential velocity of the fluid were very high. The high non-dimensional velocity exerted a violent fluctuation on the synthesis system, and high tangential velocity of the fluid could result in the creation of a whirlpool, which would lead to high concentration distribution near the side-wall. Under the co-action of gravity deposition and the whirlpool, the solid adhered to the side-wall at the bottom of the reactor. Thus, the temperature of the wall at the bottom of the reactor was higher than that of the whole synthesis system and the crystal size of the product from the bottom was larger than that from the top. 

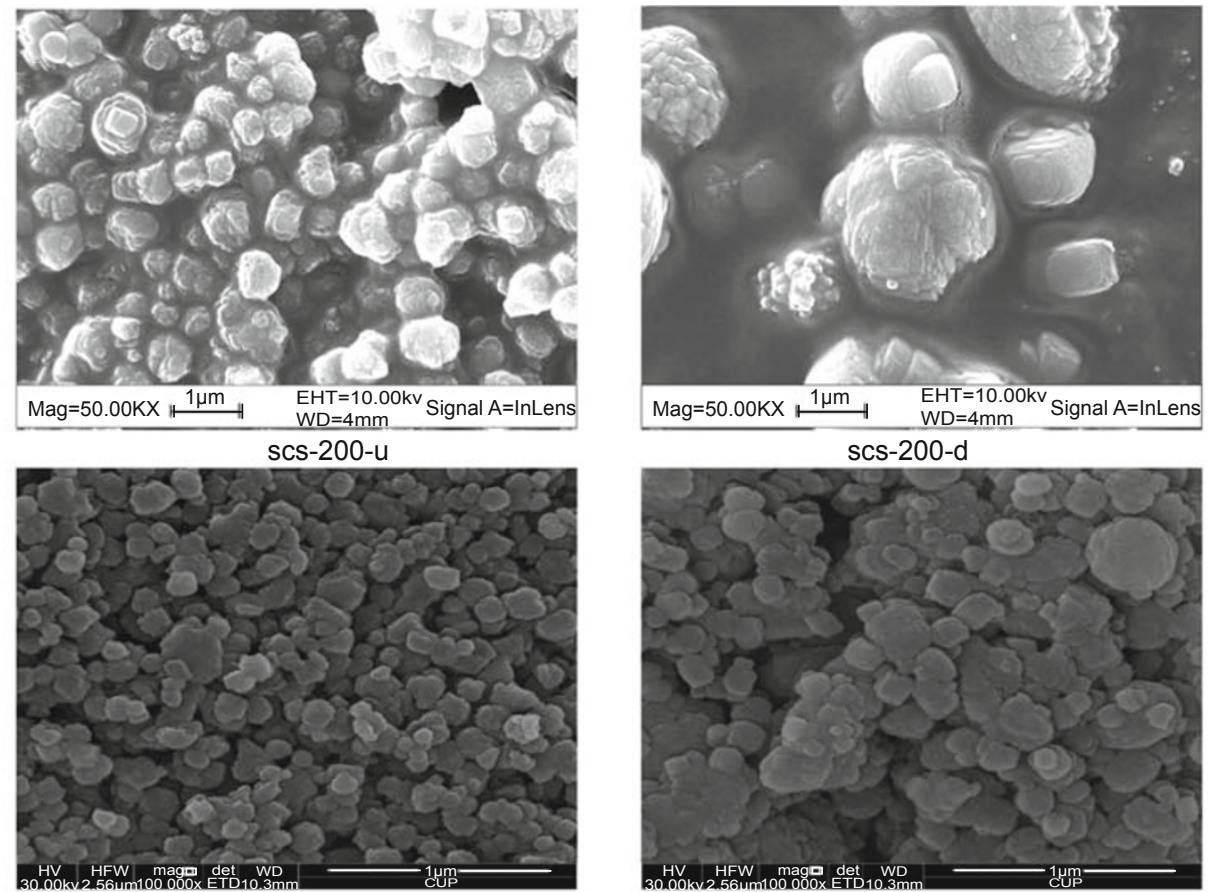

scs-300-u

scs-300-d

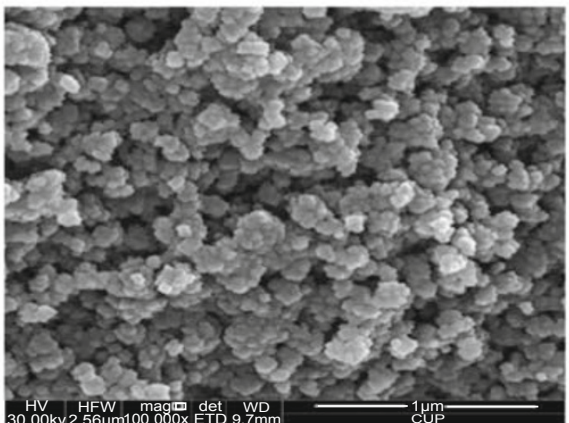

scs-400-u

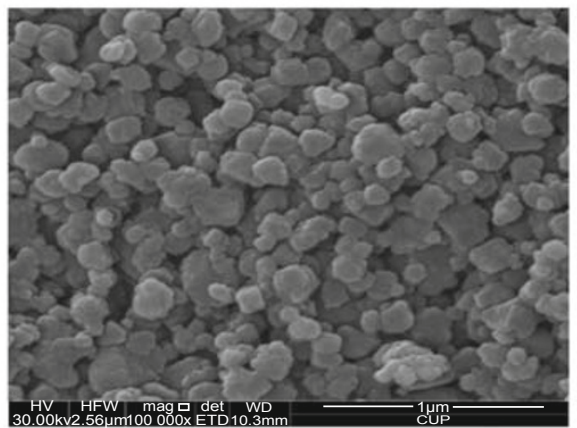

scs-500-u

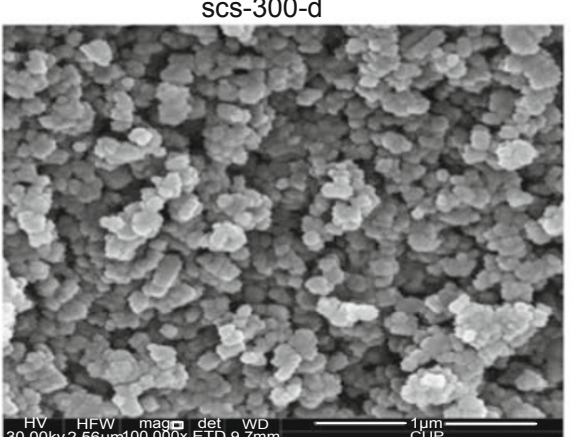

scs-400-d

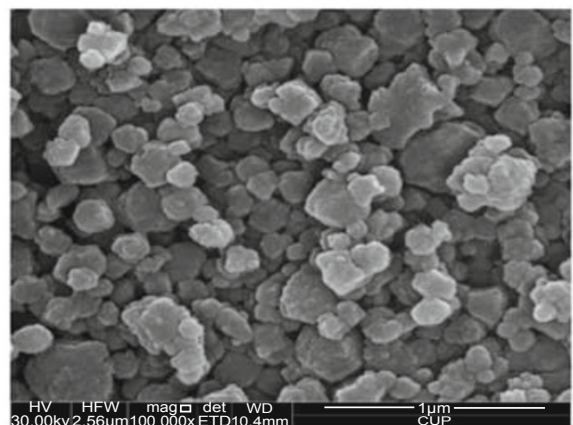

scs-500-d

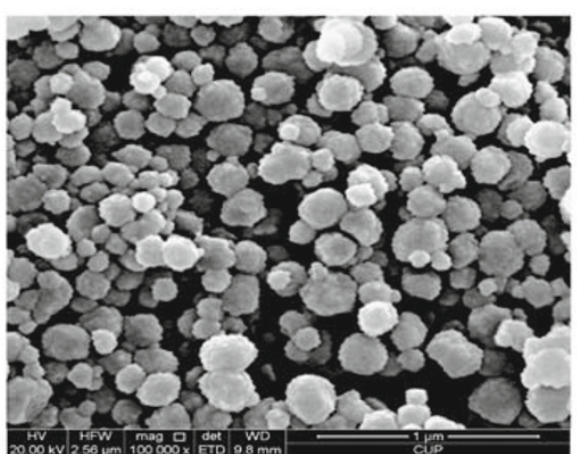

scs-600-u

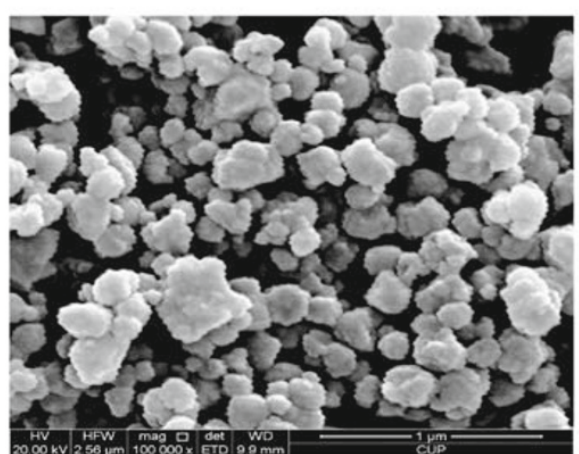

scs-600-d

Fig. 6 SEM images of the samples from the top and bottom at different stirrer speeds 


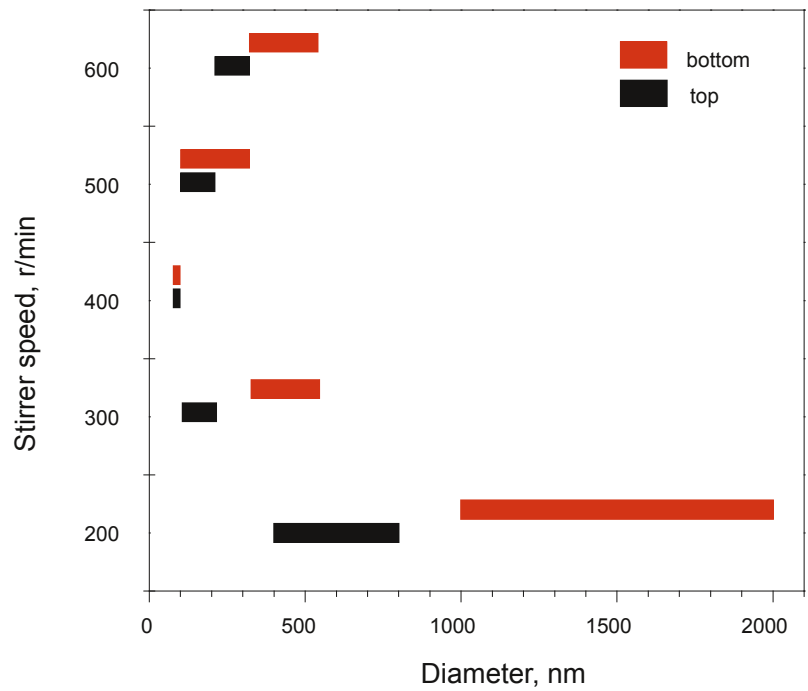

Fig. 7 Crystal size of samples from the top and bottom at different stirrer speeds

\section{Conclusions}

The work presented here shows that in the ZSM-5 zeolite crystallization process in a quasi-solid system can be controlled by tuning the flow field in the reactor. The flow field information obtained from LDV indicates that the nondimensional velocity and the tangential velocity of the fluid increase with an increase in the stirrer speed. The flow field greatly affects the heat transfer and mass transfer in the synthesis system. The morphology and size of as-synthesized of ZSM-5 in the quasi-solid system can be effectively controlled by different flow field patterns.

\section{Nomenclatures}

$C$ - Clearance from the blade to the tank

$T$ - Diameter of the stirrer tank reactor

$D$ - Diameter of the blade

$N$ - Stirrer speed

$H$ - Height of the stirrer blade

$d$-Thickness of the blade

\section{References}

Bolinder C J and Sundén B. Flow visualization and LDV measurements of laminar flow in a helical square duct with finite pitch. Experimental Thermal and Fluid Science. 1995. 11: 348-363
Casci J L. Zeolite molecular sieves: Preparation and scale-up. Microporous and Mesoporous Materials. 2005. 82: 217-226

Chem N Y and Garwood A E. Some catalytic properties of ZSM-5, a new shape selective zeolite. Journal of Catalysis. 1978. 52: 453-456

Chen J B, Liu C J, Li Y K, et al. Experimental investigation of single-phase flow in structured packing by LDV. Chinese Journal of Chemical Engineering. 2007. 15: 821-827

Dou T, Kang S J, Lei X S, et al. A method of preparing NaY molecular sieve by solid like phase conversion. China Patent, CN101190795. 2008-06 (in Chinese)

Kokotailo G T, Lawton S L, Olson D H, et al. Structure of synthetic zeolite ZSM-5. Nature. 1978. 272: 437

Minkee C, Kyungsu N, Keongnam K, et al. Stable single-unit-cell nanosheets of zeolite MFI as active and long-lived catalysts. Nature. 2009. 461: 246-249

Mohamed R M, Aly H M, El-Shahat M F et al. Effect of the silica sources on the crystallinity of nanosized ZSM-5 zeolite. Microporous and Mesoporous Materials. 2005. 79: 7-12

Sang S Y, Chang F X, Liu Z M, et al. Difference of ZSM-5 zeolites synthesized with various templates. Catalysis Today. 2004. 93: 729-734

Schäfer M, Höfken M, and Durst F, et al. Detailed LDV measurements for visualization of the flow field within a stirred-tank reactor equipped with a Rushton turbine. Chemical Engineering Research and Design. 1997. 75: 729-736

Schwingshackl C W, Massei L, Zang C, et al. A constant scanning LDV technique for cylindrical structures: Simulation and measurement. Mechanical Systems and Signal Processing. 2009. 24: 394-405

Wallenstein D and Harding R H. The dependence of ZSM-5 additive performance on the hydrogen-transfer activity of the REUSY base catalyst in fluid catalytic cracking. Applied Catalysis A: General. 2001. 214: 11-29

Wang H L and Chen Z M. System of laser Doppler's flow velocity measurement. Laser \& Infrared. 2009. 39: 721-723 (in Chinese)

Wang P, Shen B J and Gao J S. Synthesis of ZSM-5 zeolite from expanded perlite and its catalytic performance in FCC gasoline aromatization. Catalysis Today. 2007. 125: 155-162

Xie S J, Niu X L, Liu S L, et al. The scale-up of the molecular sieve of ZSM-35. Industrial Catalysis. 2006. 14: 387-389 (in Chinese)

Yamamura M, Chaki K, Wakatsuki T, et al. Synthesis of ZSM-5 zeolite with small crystal size and its catalytic performance for ethylene oligomerization. Zeolites. 1994. 14(8): 643-649

Yianneskis M, Popiolek Z and Whitelaw J H. An experimental study of the steady and unsteady flow characteristics of stirred reactors. Journal of Fluid Mechanics. 1987. 175: 537-555

(Edited by Zhu Xiuqin) 